\title{
Motivaciones hacia la práctica física deportiva y su entorno social en el alumnado de secundaria de un centro público y un centro privado de Granada
}

\section{Motivations for the physical and sport activity and their social environment in the secondary students from a public center and a private center in Granada}

\author{
Francisco José Marín García y Ana Olivares Ortega \\ Universidad de Murcia
}

Resumen: Con el trabajo aquí presente se pretende compartir cuales son las motivaciones que llevan al alumnado de ESO a realizar actividad fisica como ocupación de su tiempo libre y algunos aspectos importantes sobre las personas que forman parte de su entorno social más cercano, como por ejemplo, cual es la actitud de los padres frente a la práctica de actividad física, con quien la comparten o quienes le iniciaron. La metodología con la que hemos desarrollado nuestra investigación es cuantitativa y el método utilizado es el descriptivo. Las técnicas empleadas son la observación documental y la encuesta y dentro de ésta, el instrumento utilizado ha sido el cuestionario. Entre los resultados más interesantes obtenemos que las principales motivaciones que llevan al alumnado a practicar están relacionados con el placer que les transmite. Los amigos aparecen como el colectivo más importantes del entorno social.

Palabras clave: Motivos práctica, secundaria, tiempo libre, centro público y privado.

Abstract: The purpose of our research is to look at the motivation of secondary school students towards physical activity as a way to spend their spare time. We will consider the attitude of people who are close to them, such their parents. Ours methodology has included quantitative techniques such as questionnaire analysis plus descriptive methods including observation. The most interesting result shows that the main motivation for students to do physical activity is related to the pleasure of doing it. Friends are the most important element in the social environment.

Key words: motivation, secondary school, spare time, free time, public and private educational center.

\section{Introducción}

El trabajo aquí presente, forma parte de una investigación que ha sido llevado a cabo para descubrir si existen diferencias en los comportamientos de práctica físico-deportiva y hábitos de vida y de consumo en el alumnado de ESO entre dos centros de enseñanza, uno público y otro privado-concertado, situados en un mismo contexto. Además queremos conocer en qué grado y de qué modo influye la actitud del profesor y la asignatura de educación física en la adquisición, mantenimiento o abandono de todo este tipo de hábitos.

En este trabajo conoceremos cuales son las motivaciones que llevan al alumnado a realizar práctica de actividad física y cómo influye el entorno social, más cercano al alumnado, en los comportamientos de práctica físico deportiva.

La investigación de la que forma parte este trabajo, ha sido llevada a cabo con la elaboración de un cuestionario siguiendo los pasos pertinentes, para que cumpla con los objetivos de dicha investigación. Tras este proceso, procedimos a la recogida de datos en ambos centros. Después pasamos los datos a soporte informático y con la ayuda del programa SPSS, pudimos llevar a cabo el análisis de datos.

Deseamos que esta investigación contribuya al enriquecimiento de la base de informacióny al análisis de la situación actual de la investigación social aplicada a la práctica físico-deportiva.

\section{Objetivos}

Los objetivos que nos planteamos en nuestra investigación se detallan a continuación; debemos tener en cuenta que todos ellos han sido analizados atendiendo al tipo de centro (uno público y otro privadoconcertado) de la población objeto de estudio y al sexo:

\footnotetext{
Fecha recepción: 18-09-08 - Fecha envío revisores: 19-09-08 - Fecha de aceptación: 10-11-08 Correspondencia: Ana Olivares Ortega

C/ Argentina, $\mathrm{s} / \mathrm{n}$

30720 Santigo de la Ribera (Mrica)

E-mail: anaolivares@um.es
}

$\varnothing$ Analizar las motivaciones que inducen al alumnado de ESO a continuar, abandonar o no realizar la práctica físico y/o deportiva en su tiempo libre.

$\varnothing$ Conocer quienes han sido las personas que iniciaron en la práctica físico-deportiva al alumnado de ESO.

$\varnothing$ Descubrir con quien comparten la práctica físico deportiva el alumnado de ESO.

$\varnothing$ Estudiar cual ha sido la actitud de los padres del alumnado de ESO, en relación la actividad física deportiva practicada en su tiempo libre.

\section{Método}

La población objeto de este estudio está constituida por el alumnado de $1^{\circ}, 2^{\circ}, 3^{\circ}$ y $4^{\circ}$ de E.S.O. de dos centros de Educación Secundaria Obligatoria ubicados en un mismo contexto, pero con una diferencia entre ellos esencial para esta investigación: uno es de carácter público y el otro privado-concertado, lo que nos permitirá conocer las similitudes o contrastes en la ocupación del tiempo libre y el interés que presentan hacia la práctica físico deportiva en los sujetos de ambos centros, con un mayor grado de significatividad gracias a este factor contextual.

El número total de sujetos de nuestro estudio es de 559, de los cuales 257 alumnos/as son del público y 302 del privado. El reparto en relación al curso académico del alumnado es: 74 de $1^{\circ}$ E.S.O., 89 de $2^{\circ}$ E.S.O., 207 de $3^{\circ}$ E.S.O., 189 de $4^{\circ}$ E.S.O.

La metodología con la que hemos llevado nuestra investigación es cuantitativay el método utilizado es el descriptivo. Las técnicas empleadas son la observación documental y la encuesta, y dentro de ésta, el instrumento de recogida de datos utilizado ha sido el cuestionario.

\section{Análisis de resultados}

En este apartado comentaremos cuales son los motivos que llevan al alumnado a realizar actividad física en su tiempo libre, así como conocer las personas que le animaron o iniciaron a la práctica de actividad física deportiva. Acontinuación, analizaremos con quien la comparten y por último, cuál es la actitud de los padres en la práctica física de sus hijos/as. 

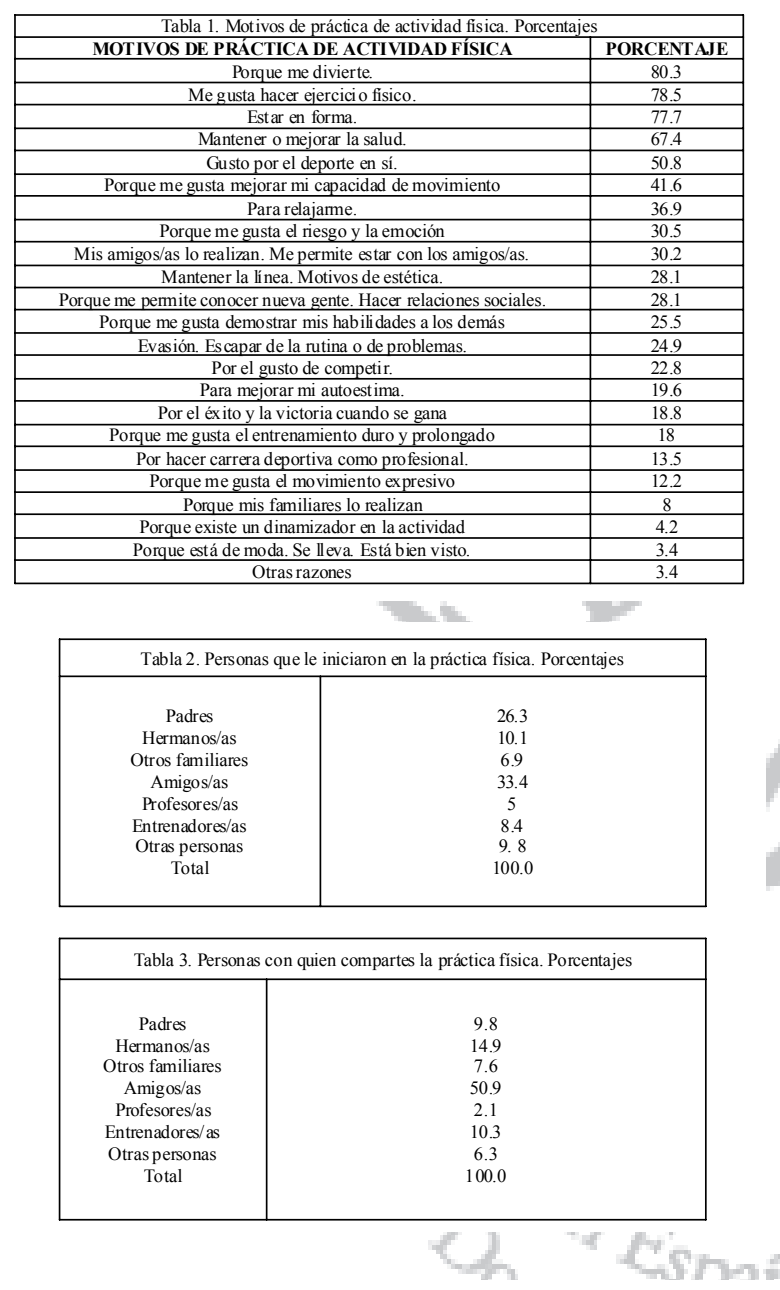

Tabla 4. Actitud de los padres en la práctica física del hijo/a. Frecuencias y Porcentajes.

\begin{tabular}{|c|c|c|c|c|}
\hline & & Frecuencia & Pacentaje & Porcentáe válido \\
\hline & $\begin{array}{l}\text { No selo ha plante ado } \\
\text { Le han pue sto }\end{array}$ & $\begin{array}{l}96 \\
9\end{array}$ & $\begin{array}{r}17,2 \\
23 \\
\end{array}$ & 25,9 \\
\hline & $\begin{array}{l}\text { impedime rtos } \\
\text { Les hasido indiferent }\end{array}$ & ${ }_{62}^{13}$ & $\begin{array}{r}2,3 \\
11,1\end{array}$ & \\
\hline & Me ran inoentivado & 175 & 31,3 & $\begin{array}{l}1,6 \\
47,3 \\
4\end{array}$ \\
\hline & Me a han impuesto & 24 & 4,3 & \\
\hline & Total & 370 & 66,2 & \\
\hline Perdidos & Sistema & 120 & 33,8 & \\
\hline Tota & & 598 & 100,0 & \\
\hline
\end{tabular}

Tabla 5. Actitud de los padres en la práctica de actividad física del hijo/a. Porcentajes y distribución $\%$ de Sexo según el sexo. Nivel de significación

\begin{tabular}{|c|r|r|r|r|r|}
\hline & \multicolumn{5}{|c|}{ Actitud de los padres en la práctica fisica del hijo/a } \\
\cline { 2 - 6 } & $\begin{array}{c}\text { No selo ha } \\
\text { planteado }\end{array}$ & $\begin{array}{c}\text { Le han puesto } \\
\text { impedimentos }\end{array}$ & $\begin{array}{c}\text { Les ha sido } \\
\text { indiferente }\end{array}$ & $\begin{array}{c}\text { Me han } \\
\text { incentivado }\end{array}$ & $\begin{array}{c}\text { Me la han } \\
\text { impuesto }\end{array}$ \\
\hline Varón & $23,4 \%$ & $4,3 \%$ & $18,7 \%$ & $44,0 \%$ & $9,6 \%$ \\
Mujer & $29,7 \%$ & $2,5 \%$ & $13,9 \%$ & $51,3 \%$ & $2,5 \%$ \\
Total & $26,2 \%$ & $3,5 \%$ & $16,6 \%$ & $47,1 \%$ & $6,5 \%$ \\
\hline
\end{tabular}

$\chi^{2}=11,198$ N.S *

Dependiendo de las variables, realizaremos un análisis descriptivo y a continuación, un análisis inferencial (tablas de contingencias) dentro de cada uno de los apartados. este último nos ayudará a conocer las diferentes manifestaciones de la población objeto de estudio, atendiendo a la variable sexo, centro y centro-sexo.

\subsection{Motivos de práctica de actividad física y su categorización}

Con la finalidad de conocer las posibles motivaciones que llevan al alumnado de E.S.O. a realizar actividad física, en el cuestionario que se les pidió que contestaran, se les facilitó una amplia lista con motivaciones de muy diversa índole (personales, estéticos, familiares,...) para que indicasen aquellas que les llevan a realizar práctica de actividad física.

Observamos que los motivos más seleccionados por la población objeto de estudio (tabla 1) que practica actividad física en su tiempo libre, son los siguientes: ocho de cada diez alumnos/as de la población afirma que continúa practicando porque le divierte hacer ejercicio físico (80.3\%), porque le gusta $(78.5 \%$ ) y para estar en forma $(77.7 \%)$.

El hecho de practicar actividad fisica para mantener o mejorar la salud, lo afirman setenta de cada cien alumnos/as, mientras que la mitad de ellos/as aseveran que practican por el deporte en sí (tabla 1).

Consideramos adecuado centrarnos en tres motivos, por la relación directa que establece con las personas que forman parte de su entorno más cercano. Alrededor de tres de cada diez alumnos/as han contestado que practica actividad fisica porque sus amigos la realizan y le facilita estar con los amigos/as. En torno a esta cifra, también encontramos que afirman porque le permite conocer nueva gente y hacer relaciones sociales. La sociabilidad en estas edades es muy importante desarrollarla para que el alumno/a evolucione hacia su formación personal lo más enriquecedora posible. Y por último, ocho de cada diez nos comunican que para ellos/as otro motivo es porque sus familiares lo realizan. (Tabla 1)

Otras motivaciones que le llevan a practicar a dos y tres de cada diez de los que han respondido, nos comentan que son: mantener la línea y motivos de estética, porque le gusta demostrar sus habilidades a los demás, por evasión y escapar de la rutina o de problemas y por el gusto de competir (tabla 1).

Las motivaciones que completan el listado obtienen porcentajes inferiores a un veinte por ciento como son: para mejorar su autoestima, por el éxito y la victoria cuando se gana, porque le gusta el entrenamiento duro y prolongado, por hacer carrera deportiva como profesional, porque le gusta el movimiento expresivo, porque existe un dinamizador en la actividad y porque está de moda (tabla 1).

4.2. Personas que animaron o iniciaron en la práctica de actividad física y deportiva

De manera descriptiva y en general, sin tener en cuenta el centro de procedencia y sexo, se puede encontrar datos interesantes en cuanto a las personas que le animaron a practicar actividad física al alumnado. Resulta atrayente observar que el mayor porcentaje se lo llevan los amigos/as con un tercio del total, ratificando el papel tan importante que supone esta figura humana para el alumnado en estas edades en desarrollo.

Los padres forman parte del entorno más cercano al alumnado, lo que se convierte en unas de las personas más influyentes sobre éstos y como aquí indica lo hace sobre la práctica de actividad física con una cuarta parte $(26.3 \%)$.

De este círculo familiar también forman parte los hermanos/as con una decena porcentual $(10 \%)$. El resto, con menos de diez unidades porcentuales, lo conforman otros familiares (6.9\%), entrenadores $(8.3 \%)$ y a destacar, el profesor/a (5\%), lo que nos ha llamado mucho la atención por el pequeño porcentaje que ocupa dentro de aquellas personas que le iniciaron en la práctica física-deportiva (tabla 2).

4.3. Personas con quien comparten sus actividades físicas o deportivas en el tiempo libre

Podemos encontrar, que cinco de cada diez sujetos que han respondido a esta pregunta en nuestro cuestionario, consideran que las personas con quien comparten sus actividades fisicas y deportivas en su tiempo libre, son los/as amigos/as (51\%), seguidos de los hermanos (15\%) y los padres $(9.8 \%)$. Tan sólo dos de cada diez, afirman compartir su tiempo con el profesorado de Educación Física en la práctica físicodeportiva (tabla 3 ).

\subsection{Actitud de los padres en la práctica física del hijo/a}

Los padres del alumnado son figuras importantes dentro de la familia, lo que ayuda reforzar actitudes positivas sobre sus hijos/as, pues la mitad de los que han contestado a nuestro cuestionario, afirman que a ellos les han incentivado sus padres. 
Un cuarto del alumnado, ratifican que no se han planteado la actitud de sus padres ante la práctica físico-deportiva. Un $16.8 \%$, afirman que la actitud de sus padres ha sido indiferente, frente al $6.56 \%$ los cuales afirman que se lo han impuesto. Tan sólo tres de cada cien alumnos/as, sus padres les hayan puesto impedimentos (tabla 4).

Si se analiza la variable sexo en relación a la actitud que tienen los padres con sus hijos/as, obtenemos diferencias estadísticamente significativas, ya que podemos observar cómo los padres han impuesto cinco veces más a sus hijos varones $(9.6 \%)$ que a sus hijas $(2.5 \%)$ la práctica de la actividad física. Consideramos que es un dato muy interesante, ya que podría ser una variable que estuviese influyendo al varón a la hora de realizar práctica de actividad física-deportiva. Por tanto, también se podría afirmar que el hecho de que sus padres impongan a la chica en menor medida a realizar actividad física-deportiva, pueda generar un menos interés y, en consecuencia, una menor práctica de ésta (tabla 5).
No existen diferencias significativas en la actitud de los padres en el centro público y privado, entre chicos y chicas a la hora de incentivarles o no hacia la práctica de actividad físico deportiva

\section{Conclusiones}

Los principales motivos por los que eligen realizar actividad física son porque se divierten y porque les gusta.

Los amigos/as ocupan un papel muy importante dentro de su entorno social. Son las principales personas que le animaron y con las que comparten la actividad física.

La mitad del total de los padres han incentivado a sus hijos, de los cuales un cuarto han animado a sus hijos y de los que escasamente una decena practican con ellos.

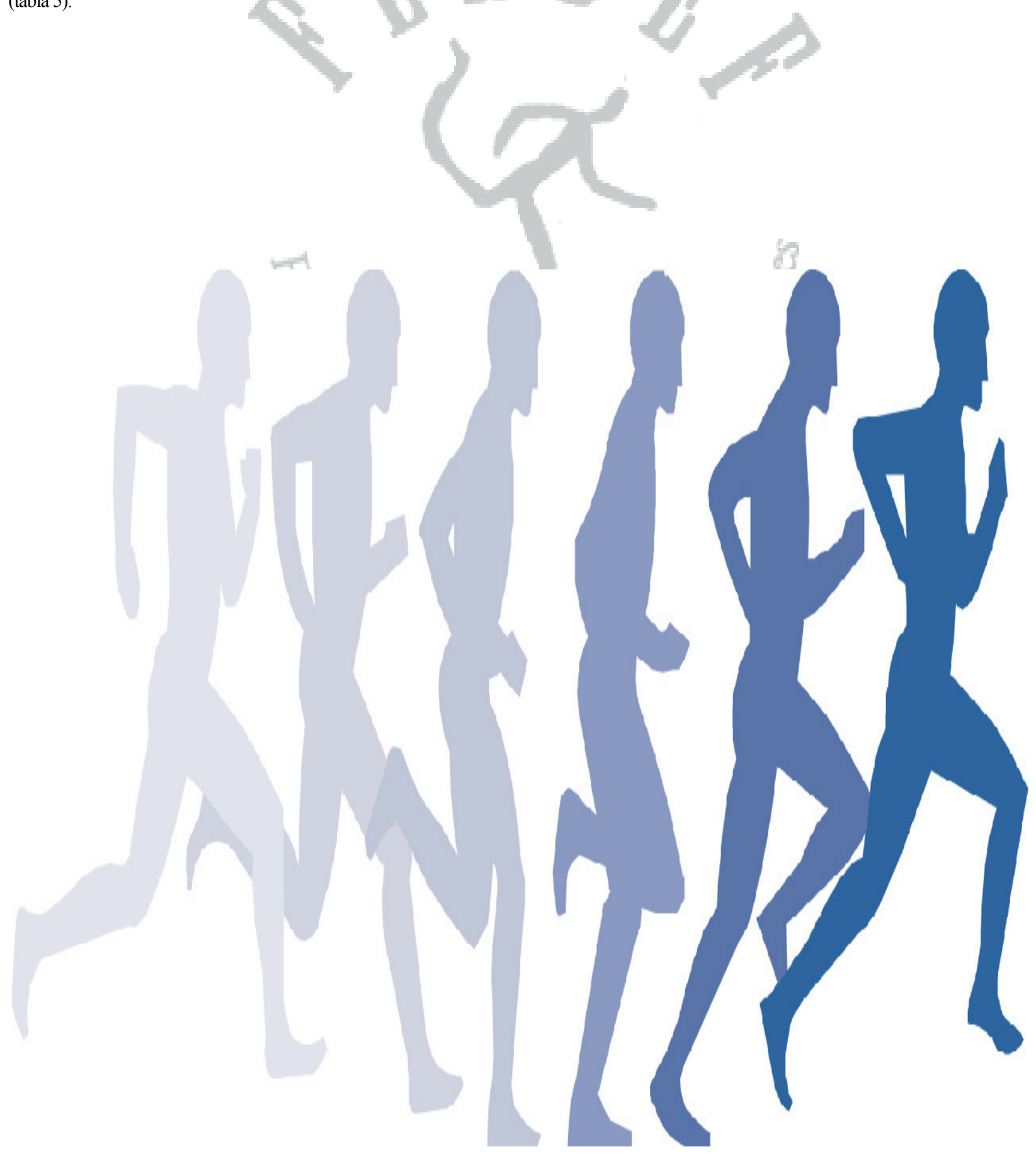

